PROPAGATION OF FIRST SOUND IN LIQUID ${ }^{3} \mathrm{He}$

G. Eska, K. Neumaier, K. Uhlig and W. Wiedemann

Zentralinstitut für Tieftemperaturforschung der Bayerischen Akademie der Wissenschaften D-8046 Garching, W.-Germany

\title{
w. Schoepe
}

Universität Regensburg, D-8400 Regensburg, W.-Germany

and

P. Wölfle

Technische Universität München, Fachbereich Physik D-8046 Garching, W.-Germany

The dispersion of first (hydrodynamic) sound in normalfluid and superfluid ${ }^{3}$ He has been measured in a cylindrical resonator at a pressure of 18.8 bar in the frequency range between $85 \mathrm{kHz}$ and $227 \mathrm{kHz}$ and in the temperature range between 1.2 and $50 \mathrm{mK}$. The scurd velocity measured in the normalfluid regime confirms recent theories of the slip effect in Fermi liquids; in superfluid ${ }^{3} \mathrm{He}-\mathrm{B}$ we find qualitative agreement between experiment and theoretical sound velocity.

\section{INTRODUCTION}

From estimates of the dispersion law of first sound in liquid ${ }^{3}$ He at low temperatures one expects a small decrease $(<0,38)$ of the sound velocity $c_{1}$ with decreasing temperature down to $T_{C}$ (the superfluid transition temperature). Our pulse method of measuring both sound attenuation and sound velocity $c_{1}$ yields a sufficient accuracy for $c_{1}$ to allow for quantitative comparisons between theory $/ 1,2 /$ and experiment.

Jensen et al. /2/ have predicted the slip effect for Fermi liquids, an effect already known from the description of rarefied gases. We present an experimental confirmation of this effectinliquid $3 \mathrm{He}$.

\section{EXPERIMENTAL}

The experimental arrangement was similar as in our earlier experiments $13 /$, but the data handling was improved and the pressure stability in the experimental cell was about 1 part in $10^{5}$.

Our experiments were performed at 5 different frequencies, for which we choose the odd harmonics from $85 \mathrm{kHz}$ to $227 \mathrm{kHz}$ ( 5 th to 13th harmonic). The frequencies were kept low in order to remain in the hydrodynamic regime. The pressure in the experimental chamber was 18.8 bar, the temperature ranged from $1.2 \mathrm{mK}$ to $50 \mathrm{mk}$.

Fig. I represents a typlcal sound velocity curve (7th harmonic, $122 \mathrm{kHz}$ ). Note that the dip of the sound velocity at $T_{C}$ is less than 0.3 percent; the other harmonics give simflar results.

To evaluate our measurements of sound dispersion we used a recently published theory $/ 1 /$ where a visco-elastic model description of the Fermi liquid was employed and which is applicable in the hydrodnamic and in the collisionless regime. There are two main contributions for the disper-

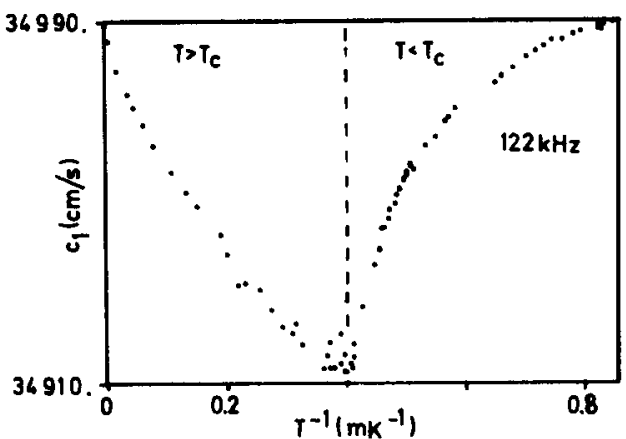

Fig. 1: Sound velocity at 18.8 bar and $122 \mathrm{kHz}$ sion relation in our case, one coming from surface friction and a smaller one from the bulk attenuation. The contribution from the bulk includes zero sound effects at higher frequencies; the contribution from the wall includes the slip effect. A slip of the quasiparticles at the walls develops then when the quasiparticle mean free path becomes comparable with the size of the viscous mean free path, which leads to an increasing contribution with decreasing temperature.

Fig. 2 shows the influence of the slip effect on the sound velocity $(227 \mathrm{kHz}, 13 \mathrm{th}$ harmonic) in the normalfluid phase.

At $\mathrm{T}_{\mathrm{c}}$ the dip of the second velocity is reduced to about 35 when compared with the hydrodynamic case $\omega T=0$ (curve a), with $\tau$ being the quasiparticle relaxation time. A correction from zero sound (c) is included in the figure. As seen from this figure we have good agreement between theory and experiment for the sound velocity; the other harmonics show very good agreement with the theoretical description, too. 


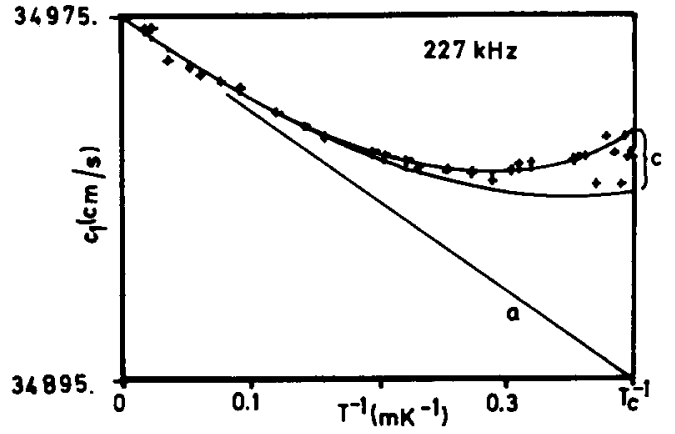

Fig. 2: Normalfluid sound velocity at $227 \mathrm{kHz}$

From the sound attenuation we derived the viscosity $\eta$. In the normalfluid phase $\eta$ agrees nicely with recently published data /4/ and our results confirm the expected $\mathrm{T}^{-2}$ behaviour of the viscosity. In the superfluid phase our earlier measurements of $n / 3 /$ were confirmed.

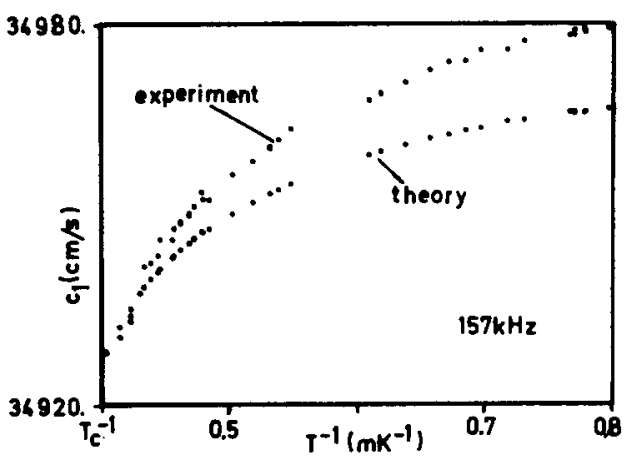

Fig. 3: Experimental and theoretical sound velocity

Fig. 3 shows the values determined experimentally for $c_{1}$ in the superfluid phase (upper curve) and $c_{1}$ as calculated after Ref. 2. The deviations at low temperatures are about $15 \mathrm{~cm} / \mathrm{s}$ whereas the experimental scatter of the points is $1.5 \mathrm{~cm} / \mathrm{s}$.

Uncertainties of the Landau parameters or the normalfluid density (as cited in Ref. 3) as well as in our temperature determination cannot explain these deviations. At the moment possible systematical errors which may be induced by our resonator geometry are being examined, but we feel they are not responsible for the deviation because of the good agreement of our data with theory in the normalfluid phase.

In the theoretical description purely diffusive scattering of the quasiparticles at the walls was assumed. If one allows for partly specular scattering as mentioned in Ref. 2 it would raise the theoretical values of $c_{1}$. The authors of Ref. 2 define the diffusivity factor (1-s); $s=0$ corresponding to purely diffusive scattering and $s=1$ to purely specular scattering, respectively. The specularity factor $\mathrm{s}$ which would be necessary to get consistency between theory and our experiment is shown in Fig. 4.

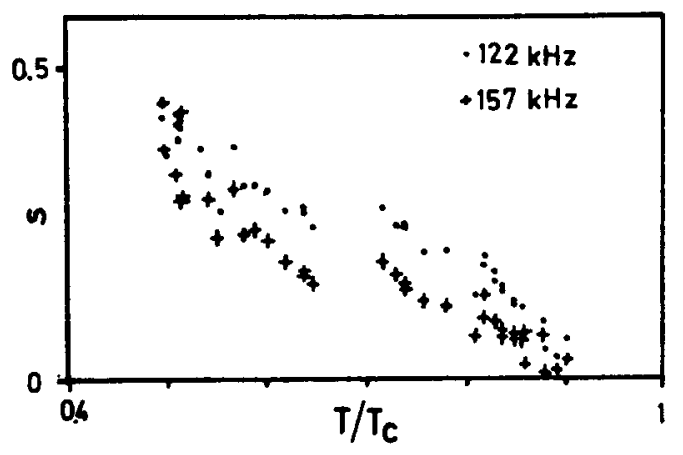

Fig. 4: Specular reflection factor

At our lowest temperatures the analysis yields a portion of specular scattering of about $45 \%$. here we have induced the data of two harmonics.

The parameter $s$ as shown above can describe our data in the superfluid phase, but a theoretical description of this parameter, especially of its temperature dependence is still lacking.

REFERENCES :

/l/ Nagai, K. and Wölfle, P., to be published

/2/ Jensen, H., Smith, H., Wölfle, P., Nagai, K., and Bisgaard, T., J. of Low Temp. Phys. 41 (1981) 473

/3/ Eska, G., Neumaier, K., Schoepe, W., Uhlig, K., Wiedemann, w. and Wölfle, P., Phys. Rev. Lett. 44 (1980) 1337

/4/ Parpia, J.M., Sandiford, D.J., Berthold, J.E. and Reppy, J.D., Phys. Rev. Lett. 40 (1978) 565 